\title{
Erratum to: Tool-use and instrumental learning in the Eurasian jay (Garrulus glandarius)
}

\author{
Lucy G. Cheke • Christopher D. Bird • \\ Nicola S. Clayton
}

Published online: 23 February 2011

(C) Springer-Verlag 2011

\section{Erratum to: Anim Cogn \\ DOI 10.1007/s10071-011-0379-4}

In the original version of the article, the words "black" and "white" in the legends of Figs. 1 and 9 had been reversed. The correct figure legends are produced below:

Fig. 1 Models of task performance given different learning heuristics. Boxes marked in white indicate tasks that would be predicted not to be passed or to take significantly longer to pass than boxes marked in black
Fig. 9 Pattern of actual task performance. White squares represent tasks in which a preference for the rewarded tube/apparatus was not apparent from total items inserted, black squares indicate a preference for the rewarded tube/apparatus, and grey squares indicate an unclear result (i.e. approached, but did not reach, significance). Dotted squares indicate tasks not performed. The order in which the experiments were undertaken is indicated by the number in the box. Here, we see that Ainsley, Hunter and Wiggins appear unable to learn, or unwilling to participate in any of the tasks, while Hoy and Romero's pattern of task performance most closely resembles the pattern of performance predicted by model $\mathrm{E}$. That is, that both instrumental conditioning and causal knowledge contribute to learning

The online version of the original article can be found under doi:10.1007/s10071-011-0379-4.

L. G. Cheke · N. S. Clayton ( $\square)$

Department of Experimental Psychology,

University of Cambridge, Cambridge, UK

e-mail: nsc22@cam.ac.uk

C. D. Bird

Sub-Department of Animal Behaviour,

University of Cambridge, Cambridge, UK 